\title{
O TESTE CLOZE DE MÚLTIPLA ESCOLHA NA AVALIAÇÃO EM COMPREENSÃO DE LEITURA NA LÍNGUA INGLESA: UM ESTUDO COM MESTRANDOS DA ÁREA DA SAÚDE
}

\author{
MULTIPLE-CHOICE CLOZE TEST IN THE EVALUATION OF ENGLISH \\ READING COMPREHESION: A STUDY WITH MASTER'S STUDENTS FROM \\ HEALTH-RELATED COURSES
}

\author{
Fernanda Goulart Ritti Dias \\ Professora no IFSP \\ Mestre em Educação pela UFAL \\ fergoulart@hotmail.com
}

Maria Inez Matoso Silveira

Professora Doutora nos Programas de Pós-Graduação em Educação e Letras da UFAL

mimatoso@uol.com.br

\begin{abstract}
RESUMO: O objetivo deste trabalho é apresentar resultados parciais de uma pesquisa de mestrado em que foi avaliada a compreensão de leitura em língua inglesa de 69 mestrandos da área de saúde por meio de um teste cloze de múltipla escolha. O teste cloze é uma técnica atualmente utilizada para avaliar a compreensão de leitura nos diversos níveis de escolaridade e sua aceitação por pesquisadores tem sido demonstrada por muitas publicações nacionais e internacionais (KLEIMAN, 1983; SANTOS 2005, 2009; OLIVEIRA 2009; BROWN, 2002, entre outros). A compreensão de leitura requerida pela técnica de cloze envolve, entre outras variáveis, a habilidade do leitor em estabelecer relações entre os elementos do texto e a sua capacidade de desenvolver associações apropriadas entre o conhecimento prévio e a informação impressa (compreensão inferencial e lexical). A possibilidade de utilizar a técnica de cloze para medir a proficiência de inglês como segunda língua é antiga e tem sido considerada por muitos um meio efetivo e prático de avaliar a compreensão de leitura. Assim, pretendemos mostrar como esse teste foi elaborado e aplicado. Também apontaremos os pontos fortes e as limitações desta técnica para avaliação de leitura em língua inglesa.

PALAVRAS-CHAVE: Teste cloze. Compreensão de leitura. Leitura e compreensão em inglês. Inglês na área de saúde.
\end{abstract}

ABSTRACT: The objective of this paper is to present partial results from a master's research aimed at assessing English reading comprehension among 69 master's students enrolled at five health-related graduate programs by means of a multiplechoice cloze test. The cloze test is used for assessing reading comprehension at several levels of education and its acceptability among researchers has been demonstrated in many national and international publications (KLEIMAN, 1983; SANTOS 2005, 2009; OLIVEIRA 2009; BROWN, 2002, among others). Reading comprehension through the cloze technique involves, among other variables, the capacity of developing appropriate associations between the previous knowledge and the printed information (inferential and lexical comprehension). The possibility of using the cloze test for measuring English as a Second Language proficiency is old and has been considered by many people an effective and practical means of assessing reading comprehension. Thus, we intend to show how this test was elaborated and applied to our participants. We will 
also show the strengths and limitations of this technique for assessing reading in English.

KEYWORDS: Cloze test. Reading comprehension. Reading and comprehension in English language. English in health-related courses.

\section{INTRODUÇÃO}

A compreensão em leitura pode ser avaliada de várias maneiras. No entanto, são poucos os instrumentos que conseguem identificar o tipo de dificuldade que o leitor pouco proficiente ou inexperiente encontra na leitura de um texto. Isso porque é difícil acessar, de forma empírica, o processamento mental-cognitivo envolvido no ato de ler, tornando complexa a avaliação da capacidade de leitura, já que os resultados dessa avaliação nem sempre podem ser observados de forma direta e objetiva (KLEIMAN, 1983). De fato, criar um instrumento confiável para mensurar a inteligibilidade ou a compreensão em leitura é algo muito complexo, pois ele deve fornecer dados relacionados não somente à capacidade de compreensão, como também à capacidade de monitorar essa compreensão.

Quando se fala em avaliar a compreensão em leitura, entende-se discriminar aqueles que têm a habilidade de ler e aqueles que não têm. Assim, qualquer teste que tenha como objetivo avaliar a leitura pode ser difícil para alguns e fácil para outros. Testar a habilidade de leitura não é apenas confirmar o que achamos que o leitor sabe, mas também revelar coisas sobre eles que não suspeitávamos.

Outro aspecto a ser considerado, e que tem sido de comum acordo entre os pesquisados da área, é que todo instrumento que visa a testar a leitura tem pontos falhos; por isso, nenhum teste consegue discriminar de forma muito precisa os comportamentos do leitor. Diante disso, fica claro que não existe um melhor método para avaliar a leitura, como reconhece Alderson (1996). Tudo depende dos objetivos do avaliador, que deverá ter em mente os aspectos a serem observados no leitor.

Um dos aspectos envolvidos na avaliação da compreensão em leitura é a classificação relacionada a bons ou maus leitores, cuja classificação remete à questão da proficiência, ou seja, leitores são geralmente classificados como proficientes ou não proficientes. Esse é, no entanto, um conceito muito complexo e controverso, pois não se pode atribuir um valor absoluto ao conceito de proficiência. Além disso, há de se considerar os graus de proficiência, o que pode gerar um resultado não confiável, visto que o critério adotado é muitas vezes subjetivo. De um modo geral, os testes que visam a avaliar essa habilidade não abrangem os diversos aspectos nela envolvidos, pois enfocam apenas uma pequena amostra do comportamento relacionado à leitura e sua compreensão (OLIVEIRA et.al., 2009).

Uma das formas mais tradicionais de avaliar a leitura é através de testes de compreensão de textos, que se utilizam de perguntas e respostas escritas. Outra forma também bastante utilizada em livros didáticos entre as décadas de 60 e 80 foram os testes de múltipla escolha. Outras duas formas que têm se mostrado eficazes na avaliação da compreensão em leitura são o protocolo verbal de leitura e o teste lacunado, mais conhecido tecnicamente como teste cloze.

Embora o teste cloze seja bem aceito por diversos pesquisadores, existem poucos estudos no Brasil que tenham utilizado a técnica para avaliar a compreensão em leitura de estudantes brasileiros na língua inglesa. Dessa forma, pretendemos, com esse 
trabalho, verificar a compreensão em leitura na língua inglesa entre mestrandos da área da saúde, utilizando, para isso, um teste cloze de múltipla escolha. Mostraremos, portanto, como o teste foi elaborado e aplicado. Também, apontaremos os pontos fortes e as limitações desta técnica para a avaliação de leitura em língua inglesa.

\section{O TESTE CLOZE}

Embora pouco utilizado no Brasil para avaliar a compreensão em leitura de estudantes na língua inglesa, o teste cloze tem sido amplamente utilizado para avaliar a leitura na língua portuguesa nos diversos níveis de escolaridade e sua aceitação por pesquisadores tem sido demonstrada em muitas publicações nacionais (CASTRO, 2008; KLEIMAN, 1983; SANTOS et.al. 2002; SANTOS, 2004; SANTOS, 2005, SANTOS et. al., 2009; SILVA; WITTER, 2008) e internacionais (ANGLAT, 2008; BRIÈRE, et.al., 1978; BROWN, 1980; 2002; MACLEAN, 1984; MCKAMEY, 2006). Para avaliação de leitura em língua inglesa como língua estrangeira, a eficácia do teste cloze já foi demonstrada por alguns autores, a exemplo de Maclean (1984), Oller (1972) e Carrel; Carson (1993)

No campo educativo, o cloze foi introduzido por Taylor em 1953, que o nomeou partindo do princípio de fechamento "closure" da psicologia de Gestalt (CUNHA, 2009). O teste consiste na organização de um texto, de mais ou menos 250 palavras, do qual se suprimem alguns vocábulos e se pede ao leitor que preencha os espaços com as palavras que melhor completarem o sentido do texto. Como proposto na sua forma original, pode ser feito um lacunamento rígido (razão fixa), no qual se apagam rigorosamente cada quinta, sétima, ou décima palavra do texto, ou um lacunamento racional, ou seja, o avaliador decide quais e quantas palavras serão omitidas. Apesar de o lacunamento rígido ser ainda o mais utilizado, alguns pesquisadores indicam o uso do lacunamento racional (MACLEAN, 1984; BROWN, 2002; BORMUTH, 1967), pois as palavras são escolhidas de acordo com os objetivos específicos do avaliador.

A compreensão em leitura requerida pelo teste cloze envolve, entre outras variáveis, a habilidade do leitor em estabelecer relações entre os elementos do texto e a sua capacidade de desenvolver associações apropriadas entre o conhecimento prévio e a informação impressa (compreensão inferencial e lexical).

Segundo Carrel e Carson (1993), o teste cloze força o leitor a usar o contexto para prever o significado e, dessa forma, parece espelhar e medir a compreensão em leitura. Leffa (1996, p. 70) afirma que, "como instrumento de ensino de leitura, o teste cloze é capaz de desenvolver no leitor a percepção de aspectos importantes do texto". Anderson (1980, apud BACHMAN, 1982), usando o procedimento racional, concluiu que o teste cloze é capaz de medir a sensibilidade de relações coesivas entre as sentenças, bem como estruturas gramaticais em nível de sentença.

$\mathrm{Na}$ medida em que os estudos sobre essa forma de avaliar a compreensão em leitura foram avançando, consolidaram-se variações do teste, que são utilizadas dependendo do objetivo pretendido, seja para diagnóstico ou remedição da compreensão em leitura (SILVA; SANTOS, 2004) "Se o pesquisador está, por exemplo, interessado em detectar determinadas estratégias usadas pelo leitor, ele poderá tentar adaptar o cloze de modo a refletir o uso ou não dessas estratégias", assim, o pesquisador pode obter resultados mais satisfatórios se controlar o lacunamento e escolher quais palavras apagar (LEFFA, 1996, p. 71). Como afirma Bormuth (1967), as palavras excluídas no teste cloze devem 
visar a um objetivo específico, do contrário, ele se transforma num teste comum de completar.

Nesse sentido, Oliveira et al. (2009) apresentam 10 tipos diferentes do teste, que, segundo as autoras, podem ser organizados dependendo do objetivo de leitura e do grau de dificuldade que se pretende atingir: 1) cloze lexical: itens lexicais, como substantivos, adjetivos, advérbios, entre outros, são omitidos; 2) cloze gramatical: itens relacionais, como verbos, conjunções, artigos, entre outros, são omitidos; 3) cloze de múltipla escolha: alternativas para o preenchimento da lacuna, dentre as quais deve-se escolher apenas uma, são oferecidas ao leitor; 4) cloze cumulativo: uma única palavra, a qual o leitor terá que identificar, é omitida sistematicamente; 5) cloze labirinto: palavras que completam a oração (a partir de três opções previamente selecionadas), devem ser elegidas pelo leitor; 6) cloze pareado: cinco palavras por oração, dispostas em um quadro ao lado da oração a qual pertencem, são omitidas. O leitor deverá eleger a palavra correta que foi omitida e completar, sendo que todas as palavras dispostas no quadro completam de forma correta alguma sentença. O tamanho do espaço é uma pista; 7) cloze restringido: todas as palavras omitidas são dispostas ao lado ou abaixo do texto. O leitor deverá escolher a palavra correta para cada espaço, sendo que uma palavra, uma vez utilizada, não poderá ser usada novamente, a menos que o quadro seja composto de duas ou mais palavras iguais; 8) Cloze com chaves de apoio: $\mathrm{O}$ risco contínuo do tamanho da palavra omitida é substituído por pequenos riscos que representam cada letra omitida; 9) Cloze pós-leitura oral: a organização desse tipo de teste é igual a do cloze tradicional, a única diferença é que, antes de iniciar o preenchimento das lacunas, o leitor deve fazer uma leitura oral de todo o texto para então começar. $\mathrm{O}$ aluno, individualmente, é orientado a realizar a leitura, e, em seguida, deverá preencher as lacunas; 10) Cloze interativo: para cada omissão, os alunos devem eleger uma palavra adequada ao contexto e justificar sua escolha. Nesse modelo de aplicação, sugere-se que o avaliador anote as justificativas do aluno.

Dentre os tipos de teste citados acima, o teste cloze de múltipla escolha é um dos que têm sido mais utilizados nas provas de proficiência em língua estrangeira, principalmente na língua inglesa, como os exames de renome, como o $\mathrm{TOEFL}^{1}$, por exemplo. O teste cloze de múltipla escolha facilita a correção e pode, dessa forma, ser aplicado a um número maior de sujeitos. Consiste em lacunar o texto e apresentar algumas sugestões ao leitor, conhecidos como distratores. O leitor deve escolher a palavra que mais se enquadra no sentido do texto. Podem ser omitidas palavras lexicais ou gramaticais, dependendo do que se pretenda avaliar.

Diferentes tipos de teste cloze mensuram diferentes habilidades ou, pelo menos, fornecem resultados diferentes uns dos outros. Contudo, é possível obter diagnósticos bastante úteis através das respostas dos alunos nesses testes (ALDERSON, 1996).

Independentemente do tipo, as vantagens incluem o seu fácil preparo e aplicação, além de seus resultados apresentarem alta correlação com os resultados de testes convencionais de leitura (i.e. testes que apresentam questões abertas e exigem que o leitor responda perguntas sobre o texto) (KLEIMAN, 1983). Além disso, como o teste é o próprio texto, não apresenta o viés dos questionários, nos quais é sempre possível

\footnotetext{
${ }^{1}$ O TOEFL (Test of English as a Foreign Language) é um exame de avaliação de proficiência em inglês para falantes não nativos, sendo um dos testes mais reconhecidos internacionalmente. Tem como objetivo avaliar a proficiência em inglês daqueles que pretendem ingressar em universidades dos Estados Unidos ou Canadá.
} 
criar itens difíceis sobre passagens fáceis e vice-versa, ou ainda criar questões não dependentes da passagem (CAPARROTI, 2005).

Quanto à pontuação dos testes cloze de razão fixa, os escores podem ser obtidos somando-se os números de lacunas preenchidas corretamente, ou pode ser baseado na correção na qual se aceita como correta uma palavra sinônima ou outra palavra adequada ao contexto da palavra eliminada. Para ambos os casos, palavras literais ou sinônimas, não se tem observado diferenças estatisticamente significativas entre os dois tipos de correção em estudos brasileiros (CAPARROTI, 2005). Porém, segundo Oller (1972), o critério de pontuação de palavras exatas pode tornar-se muito difícil para falantes não nativos.

A possibilidade de utilizar o teste cloze para medir a proficiência em inglês como segunda língua (ISL) é antiga e tem sido considerada por muitos pesquisadores um meio efetivo de avaliar proficiência em segunda língua. Lange e Clausins (1981, p. 255) afirmam que vários estudos encontraram correlações significativas entre os escores obtidos no teste cloze e outras medidas de avaliação de proficiência em língua estrangeira. Esses escores correlacionaram bem com uma variedade de medidas, entre elas fatores cognitivos, tais como: argumentação, habilidade verbal, escrita e medidas de fluência expressiva.

Carrel e Carson (1993) afirmam que, devido à capacidade do teste de medir a compreensão além do contexto imediato do item omitido, ele tem se tornado uma ferramenta importante para os pesquisadores em segunda língua para investigar as semelhanças e diferenças entre o desempenho de leitura em língua materna e língua estrangeira.

Alguns autores já se preocuparam em verificar se o teste cloze era capaz de nivelar estudantes de línguas estrangeiras de diferentes níveis. Brière et.al. (1978) avaliaram falantes nativos de inglês cursando três semestres distintos em quatro línguas: alemão, japonês, russo e espanhol, por meio de um teste cloze de aproximadamente 500 palavras para cada língua, no qual toda sétima palavra foi omitida. A partir dos resultados obtidos, puderam concluir que o teste cloze conseguiu discriminar aprendizes de línguas estrangeiras por níveis instrucionais.

Heilenman (1983), também preocupada com a falta de um recurso que conseguisse nivelar estudantes com diferentes níveis que ingressam em cursos de línguas, realizou dois experimentos. No primeiro experimento, avaliou 16 estudantes falantes nativos de inglês que eram estudantes de francês utilizando uma passagem de 255 palavras, na qual cada sétima palavra foi omitida. Os alunos realizaram, para fins de comparação, dois testes de nivelamento oferecidos regularmente na universidade, um de leitura e outro de escrita. Os resultados apontaram que os testes regulares, bem como o teste cloze, avaliaram praticamente a mesma coisa, e que o teste cloze poderia ser usado para ranquear esses estudantes de forma tão precisa quanto os outros tipos de testes, que são, na maioria das vezes, mais longos e difíceis de avaliar. O segundo experimento foi realizado com 388 alunos matriculados no primeiro, segundo e terceiro ano de francês. Os 11 professores de francês (dentre eles 6 nativos e 5 não nativos proficientes) também foram convidados para realizar o teste. $\mathrm{O}$ mesmo teste cloze do experimento foi aplicado. Os resultados mostraram que o teste cloze foi capaz de discriminar os grupos, principalmente os de mais alto nível. No entanto, a autora alerta que se deve ter cuidado ao nivelar estudantes de níveis muito próximos. 
Bensoussan e Ramraz (1984) pretenderam oferecer evidências estatísticas para a eficácia do teste cloze de múltipla escolha, bem como compará-lo com testes convencionais de múltipla escolha. Para tanto, realizaram quatro experimentos com estudantes não falantes de língua inglesa. Os resultados indicaram que, em termos de dificuldade, os testes cloze podem ser equiparados aos testes convencionais e que a escolha do texto pode ser mais relevante do que o formato do teste em si. Os resultados apontaram que o teste cloze de múltipla escolha também é capaz de avaliar a compreensão em leitura em nível macro, ou seja, a habilidade de seguir uma sequência de pensamento lógico. Segundo as autoras, o teste cloze de múltipla escolha é uma alternativa eficaz para o nivelamento de estudantes em cursos de línguas, pois, além de ser capaz de avaliar uma variedade de itens em um tempo menor, também é mais fácil de ser elaborado do que os testes convencionais de múltipla escolha.

Ao procurar avaliar a compreensão em leitura utilizando o teste cloze há que se analisarem bem os objetivos de cada pesquisador e, dependendo do caso, é interessante que se aplique mais de um tipo de teste, como, por exemplo, os protocolos verbais, que serão discutidos a seguir.

\subsection{Procedimentos metodológicos}

Esta pesquisa volta-se ao processamento cognitivo da compreensão em leitura na língua inglesa entre mestrandos da área da saúde. A opção por mestrandos como informantes desta pesquisa se deu pela grande relevância que a língua inglesa tem nesse nível acadêmico institucional (IGLESIAS; BATISTA, 2010). A pesquisa abrangeu estudantes de cinco cursos de Mestrado das áreas da saúde de uma universidade pública do estado de Pernambuco, Nordeste do Brasil, a saber: Ciências da Saúde, Biologia Molecular e Celular, Enfermagem, Educação Física e Hebiatria.

Este artigo, que faz parte de uma pesquisa maior de mestrado, mostrará os resultados provenientes da primeira etapa da pesquisa, em que participaram 69 mestrandos, sendo 19 das Ciências da Saúde, 7 de Biologia Molecular e Celular, 8 de Enfermagem, 20 de Educação Física e 15 de Hebiatria.

No primeiro contato com os pós-graduandos, os objetivos da pesquisa foram descritos, e aqueles que aceitaram participar voluntariamente da pesquisa assinaram o Termo de Consentimento Livre e Esclarecido (Registro CEP/UPE 112/11 - Registro CAAE: 0092.0.097.000-11).

A etapa que será descrita tratará dos resultados obtidos a partir da aplicação de um teste cloze de múltipla escolha em língua inglesa. No entanto, essa etapa também foi composta de um questionário de sondagem e um teste cloze em língua portuguesa. $\mathrm{O}$ questionário foi composto por 32 questões objetivas para verificar a familiaridade dos informantes com a língua inglesa bem como suas práticas e habilidades de leitura. Para tanto, o informante deveria assinalar a melhor alternativa para cada questão e caso desejasse fornecer comentários adicionais, alguns espaços em branco foram deixados em algumas respostas.

O teste cloze de múltipla escolha em português foi elaborado a partir de uma introdução de um artigo científico retirado da revista Arquivos Brasileiros de Endocrinologia \& Metabologia, apresentou 246 palavras e 28 lacunas em branco. A aplicação do teste envolveu uma instrução inicial indicando que o informante deveria primeiramente ler o 
texto todo, e, depois, voltar ao início para preencher cada lacuna com a palavra que julgasse mais adequada ao contexto. Para tanto, ele deveria escolher dentre as três opções (distratores) dispostas abaixo de cada lacuna. Como sugere a metodologia do teste, a primeira e a última frases do texto não fizeram parte do processo de lacunamento.

Para o teste cloze de múltipla escolha em inglês foi selecionada a introdução de um artigo científico, retirado de uma revista especializada da área - Medicine \& Science in Sports \& Exercise - que apresentou 304 palavras e 26 lacunas. Optamos pelo teste cloze racional $^{2}$, pois estávamos interessadas em retirar apenas palavras que julgássemos essenciais para o nosso propósito. O título foi retirado do texto e colocado abaixo, juntamente com a fonte. O critério para a escolha dos espaços em branco foram os recomendados por Bensoussan e Ramraz (1984), segundo os quais o avaliador deve escolher itens relacionados a palavras-chave em um argumento lógico para que seja possível verificar se o leitor consegue seguir a sequência do pensamento.

Visto que o nosso objetivo era testar uma variedade de palavras, removemos do texto aquelas pertencentes a classes gramaticais diversas: substantivos, adjetivos, verbos, conjunções e preposições. Como distratores, incluímos, em sua maioria, antônimos, como forma de avaliar o entendimento do texto de forma geral. Ainda, seguindo a recomendação de Bensoussan e Ramraz (1984), optamos por não fazer uso de sinônimos para os distratores, visto que os mesmos tornam as questões ambíguas, mesmo para falantes nativos.

A aplicação do teste envolveu uma instrução inicial, indicando que o informante deveria primeiramente ler todo o texto, e, depois, voltar ao início para preencher cada lacuna com a palavra que julgasse mais adequada ao contexto. Para tanto, ele deveria escolher dentre as três opções (distratores) dispostas abaixo de cada lacuna. Como sugere a metodologia do teste, a primeira e a última frase do texto não fizeram parte do processo de lacunamento.

Figura 1. Teste cloze de múltipla escolha aplicado ${ }^{3}$

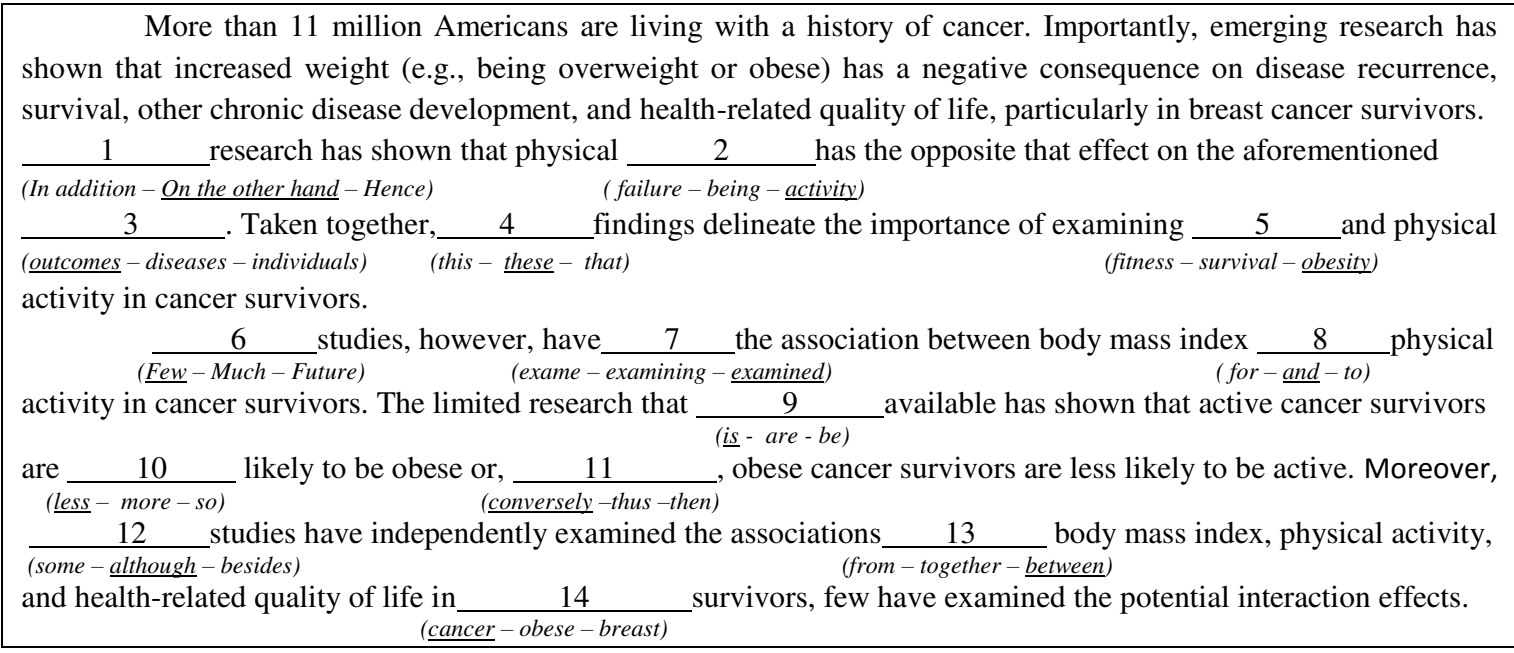

\footnotetext{
${ }^{2}$ No teste cloze racional, o pesquisador decide quais palavras farão parte do processo de lacunamento.

${ }^{3}$ Para fins de análise e melhor visualização, os números das 26 lacunas foram inseridos e a resposta correta foi grifada.
} 


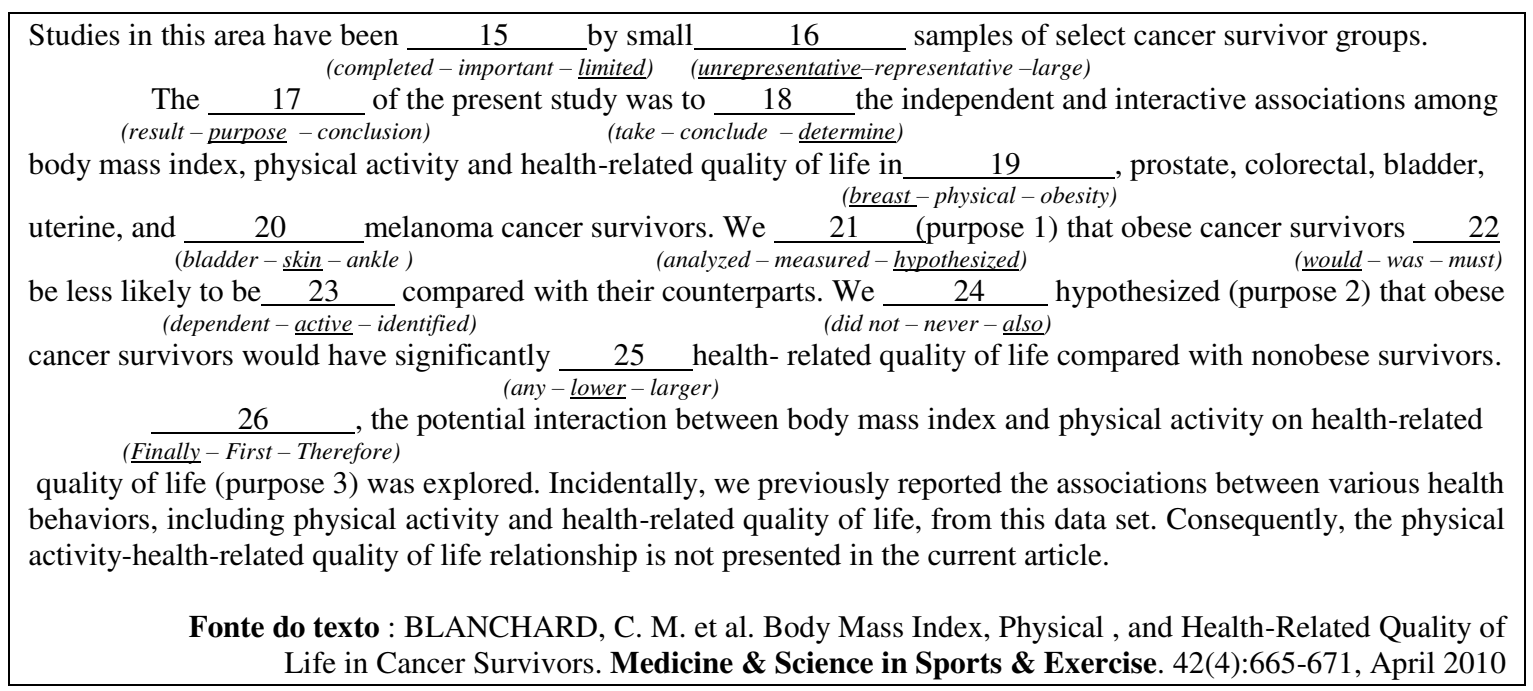

O teste cloze foi tabulado tomando como certas as palavras que se encontravam no texto original. $\mathrm{O}$ escore final correspondeu à soma das lacunas preenchidas corretamente, ou seja, a pontuação variou de 0 a 26 .

\subsection{Resultados}

Para melhor ilustrar o desempenho dos informantes, apresentaremos, na Tabela 1, as pontuações de acertos obtidas no teste cloze. Considerando-se as 26 lacunas presentes no teste, o aluno que pontuou mais fez 25 acertos, enquanto o que obteve menor número de acertos pontuou 5 .

Tabela 1. Desempenho dos 69 informantes no teste cloze aplicado.

\begin{tabular}{lcc}
\hline \multicolumn{1}{c}{ Pontuação (\%) no teste cloze } & $\begin{array}{c}\text { Número de } \\
\text { informantes }\end{array}$ & \% informantes \\
\hline 80 a $100 \%$ (21 a 26 pontos) & 20 & $29 \%$ \\
60 a 79\% (16 a 20 pontos) & 15 & $22 \%$ \\
40 a 59\% (11 a 15 pontos) & 23 & $33,5 \%$ \\
20 a 39\% (6 a 10 pontos) & 10 & $14,5 \%$ \\
0 a 19\% (0 a 5 pontos) & 1 & $1 \%$ \\
\hline
\end{tabular}

É possível notar, a partir da tabela acima, que quase a metade dos informantes (34 estudantes) obteve pontuações abaixo de 60\%. Dos 69 informantes, apenas 20 obtiveram pontuações acima de $80 \%$ no teste. Esses dados revelaram que a compreensão em leitura do texto científico em língua inglesa pode ser considerada baixa no teste aplicado.

Interessante ressaltar que o número de informantes, ou seja, 35 alunos, com as maiores pontuações (acima de $60 \%$ de acertos) está coerente com o número de informantes que afirmaram, no questionário aplicado antes do teste, utilizar a língua inglesa como principal idioma para suas pesquisas acadêmicas (cerca de 39 alunos). Também, vale frisar que esses dados estão em conformidade com o número de informantes (cerca de 
36) que relataram, também no questionário aplicado, utilizar a base de dados Pubmed, que dispõe artigos científicos em língua inglesa.

Como é possível observar na Tabela 2, dentre as lacunas que apresentaram maiores índices de erros, três estavam relacionadas aos marcadores discursivos (although, on the other hand e conversely). Assim, é possível inferir que os informantes da nossa pesquisa apresentam grande dificuldade para compreender algumas funções semânticodiscursivas dos conectivos interfrasais. Esse resultado pode apontar uma pouca familiaridade dos informantes com a leitura de textos científicos, uma vez que os conectivos são muito frequentes nos gêneros expositivo-argumentativos, como é o caso dos artigos científicos, aqui analisado.

Ainda sobre a questão da familiaridade com esse gênero acadêmico tão importante para os cursos de mestrado da área da saúde, é possível observar, a partir da análise da lacuna 17, que esses informantes parecem, de fato, não possuir domínio adequado da organização retórica da introdução de artigos científicos. Ademais, parece que os informantes também não utilizaram estratégias de leitura suficientes para completar essa lacuna uma vez que a palavra purpose se repetia no mesmo parágrafo.

Da mesma forma, na lacuna 21, para chegar à resposta correta (hypothesized), os informantes poderiam ter identificado a repetição do vocábulo na frase seguinte, na qual o autor traz a segunda hipótese do estudo. Também, fazia-se necessário reconhecer que, na introdução do artigo científico, a hipótese é geralmente trazida após a apresentação dos objetivos.

De um modo geral, os baixos resultados no teste cloze foram provenientes da falta de competência linguística, mais especificamente a lexical, que mostrou ser a maior limitação dos informantes pesquisados. A partir da análise das lacunas com maiores índices de erros, como por exemplo, a lacuna 3, fica claro que esses informantes pareceram não ter familiaridade com a palavra outcomes, que é bastante recorrente em textos científicos. Também, na lacuna 19, com quase 50\% de erros, ficou claro que os informantes não conheciam o significado da palavra breast. Nem mesmo as palavras próximas como prostate, colorectal, bladder e uterine, que eram palavras cognatas, conseguiram ajudá-los a preencher corretamente a lacuna. Caso semelhante não ocorreu com a palavra skin, que foi completada corretamente $75,4 \%$ dos participantes. Certamente, o conhecimento do vocábulo, aliado ao uso da estratégia de inferência da palavra seguinte, melanoma, contribuíram para o elevado número de acertos dessa lacuna.

Por outro lado, é interessante observar que as lacunas que tiveram menores índices de erros deveriam ser completadas com palavras cognatas, como nas lacunas 5, 18, 26, 2 e 14 (obesity, determine, finally, activity e cancer, respectivamente).

Ainda, com relação aos cognatos, o que nos chamou a atenção foi o fato de mais de a metade dos informantes não terem completado corretamente a lacuna 16. Possivelmente, o não conhecimento do prefixo un- em unrepresentative, dificultou a leitura da palavra cognata representative, ocasionando o elevado índice de erros nessa lacuna.

Tabela 2. Porcentagem de erros em cada lacuna 


\begin{tabular}{|c|c|c|}
\hline $\begin{array}{c}\text { Número da } \\
\text { lacuna }\end{array}$ & Palavra correta & $\begin{array}{c}\text { Porcentagem }(\%) \text { de } \\
\text { erros }\end{array}$ \\
\hline 12 & although & 87 \\
\hline 3 & outcomes & 76,8 \\
\hline 1 & on the other hand & 65,2 \\
\hline 10 & less & 60,9 \\
\hline 11 & conversely & 52,2 \\
\hline 16 & unrepresentative & 52,2 \\
\hline 19 & breast & 49,3 \\
\hline 17 & purpose & 43,5 \\
\hline 21 & hypothesized & 43,5 \\
\hline 25 & lower & 43,4 \\
\hline 15 & limited & 42 \\
\hline 22 & would & 42 \\
\hline 4 & these & 40,6 \\
\hline 23 & active & 39,1 \\
\hline 6 & few & 37,7 \\
\hline 5 & obesity & 33,3 \\
\hline 24 & also & 33,3 \\
\hline 9 & is & 31,9 \\
\hline 7 & examined & 27,5 \\
\hline 20 & skin & 24,6 \\
\hline 18 & determine & 23,2 \\
\hline 26 & finally & 23,2 \\
\hline 13 & between & 18,8 \\
\hline 8 & and & 15,9 \\
\hline 2 & activity & 14,5 \\
\hline 14 & cancer & 10,1 \\
\hline
\end{tabular}

É importante mencionar que após a aplicação do teste cloze, selecionamos 13 informantes com pontuações variadas para realizar o protocolo verbal de leitura, que teve como objetivo confirmar os resultados obtidos no teste cloze. O protocolo foi elaborado utilizando o mesmo texto utilizado no teste cloze. Nessa etapa, o informante explicou, oralmente e detalhadamente, o porquê da escolha de cada palavra, incluindo qualquer pista, trecho, palavra, tudo o que o fazia chegar à resposta que estava escolhendo no momento da leitura.

Dessa forma, além de aprofundar a análise dos dados obtidos por meio do teste cloze, confirmamos as principais dificuldades dos nossos informantes durante a leitura de texto científico em língua inglesa e comprovamos a eficácia do teste cloze na avaliação de leitura.

\section{CONSIDERAÇÕES FINAIS}

Diante dos resultados apresentados e analisados pudemos constatar que a grande maioria dos informantes da nossa pesquisa não possuíram habilidades de leitura 
suficientes para completar o teste cloze de múltipla escolha aplicado. Isso porque metade dos nossos informantes não conseguiram acima de $60 \%$ de acertos no teste. Sendo assim, podemos inferir que grande parte dos alunos mestrandos da área da saúde possuem compreensão em leitura na língua inglesa insuficiente para a leitura de textos acadêmico-científicos, mais especificamente artigo científico.

Dentre as lacunas com maiores índices de erros encontravam-se os marcadores discursivos, ou conectivos, responsáveis pela coesão textual e muito frequentes em textos científicos. Ademais, notamos que o vocabulário geralmente comum em textos da área não era conhecido pela maioria dos participantes, o que também evidenciou a pouca familiaridade com a organização retórica da seção introdução do artigo científico.

Diante dos resultados apresentados e analisados, foi possível verificar que o teste cloze é um instrumento confiável para avaliar a compreensão de leitura em língua inglesa. Isso foi confirmado pela aplicação de um segundo instrumento (protocolo verbal de leitura), que, além de corroborar os dados coletados no cloze, nos forneceu indicativos mais precisos quanto às dificuldades dos alunos em cada lacuna.

Para finalizar, destacamos que a construção de um teste cloze de múltipla escolha não é tarefa fácil e que devem ser levados em consideração vários critérios no momento de sua elaboração, como a seleção do texto, das palavras que serão eliminadas e, principalmente, a escolha dos distratores. Essa última é essencial para que o avaliador consiga dados mais precisos para atender aos seus objetivos que procura com o teste. Por ser um instrumento de fácil aplicação e correção, ele se mostra interessante para estudos envolvendo muitos participantes, como foi o caso desta pesquisa.

\section{REFERÊNCIAS}

ALDERSON, J. C. Reading in a foreign language: a reading problem or a language problem? In: ALDERSON, J. C.; URQUHART, A. H. Reading in a Foreign

Language. London: Longman, 1984. p.1-24.

ALDERSON, J. C. The testing of reading. In: NUTTAL, C. Teaching reading skills in a foreign language. Oxford: Heinemann, 1996.

ANGLAT, H. D. El test cloze en la evaluación de la comprensión del texto informativo de nível universitário. Revista de Lingüística Teórica y Aplicada Concepción, v. 46, n.1, p. 121-137, 2008.

BACHMAN, L. F. The trait structure of cloze test score. Tesol Quarterly v.16, n. 1, p. 61-80. 1982.

BENSOUSSAN, M.; RAMRAZ, R. Testing EFL Reading comprehension using a multiple-choice rational cloze. The Modern Language Journal, v. 68, n. 3, p. 230 239, 1984.

BORMUTH, J. R. Cloze readability procedure. Los Angeles: Universidade of California. CSEIP Occasional Report, n.1. 1967. Disponível em: < http://www.cse.ucla.edu/products/reports/R004 > Acesso em 28.04.2011. 
BRIÈRE, E. J.; CLAUSING, G.; SENKO, D.; PURCELL, E. A look at cloze testing across languages and levels source: The Modern Language Journal, v. 62, n. 1/2, p. 23-26, 1978.

BROWN, J. D. Relative merits of four methods for scoring cloze tests. The Modern Language Journal, v. 64, n. 3, p. 311-317, 1980.

BROWN, J. D. Do cloze tests work? Or, is it just an illusion? Second Language Studies, v. 21, n. 1, p. 79-125, 2002.

CAPARROTI, N. B. Prova de compreensão em leitura: evidências de validade. Itatiba 2005. Dissertação (Mestrado em Psicologia). Programa de Pós- Graduação Stricto Sensu em Psicologia.Universidade São Francisco, 2005.

CARREL, P. L; CARSON, J. G. First and second language reading strategies: evidence from cloze. Reading in a Foreign Language. v. 10, n. 1, p. 953- 965, 1993.

CASTRO, R. M. C. A. Compreensão da leitura: aplicação da técnica do procedimento close nos ensinos fundamental e médio. Crátilo: Revista de Estudos Lingüísticos e Literários. Patos de Minas, v. 1, p. 70-78, 2008.

CUNHA, N. B. Pesquisas com o teste de cloze no Brasil. In: SANTOS, A. A. A; BORUCHOVITCH, E; OLIVEIRA, K. L (Org.) Cloze: Um instrumento de diagnóstico e intervenção. São Paulo: Casa do Psicólogo, 2009, p. 79-117.

HEILENMAN, L. K. The use of a cloze procedure in foreign language placement. The Modern Language Journal, v.67, n. 2, p. 121-126, 1983.

IGLESIAS, S. R. A.; BATISTA, N. A. A língua inglesa e a formação de mestres e doutores na área da saúde. Revista Brasileira de Educação Médica, v. 34, n. 1, p. 74 $81,2010$.

KLEIMAN, A. B. Diagnóstico de dificuldades na leitura: uma proposta de instrumento. Cadernos PUC, n. 16, p. 34-50, 1983.

LANGE, D. L.; Clausing, G. An examination of two methods of generating and scoring cloze tests with students of German on three levels. The Modern Language Journal, v. 65 , n. 3, p. 254-261, 1981.

LEFFA, V. J. Aspectos da leitura. Porto Alegre: Sagra, 1996.

MACLEAN, M. Using rational cloze for diagnostic testing in L1 and L2 reading. Tesl Canada Journal/Revue Tesl du Canada, v 2, n. 1, 1984.

MCKAMEY, T. Getting closure on cloze: a validation study of the "rational deletion" method. Second Language Studies, v.24, n.2, p. 114-164, 2006.

OLIVEIRA, K.; BORUCHOVITCH, E; SANTOS, A. A. A .A técnica de Cloze na avaliação da compreensão em leitura. In: SANTOS, A. A. A; BORUCHOVITCH, E; OLIVEIRA, K. L (Org.) In: Cloze: Um instrumento de diagnóstico e intervenção. São Paulo: Casa do Psicólogo, 2009. p.47-78 
OLLER, J. W. Scoring methods and difficulty levels for cloze tests of proficiency in English as a second language. The Modern Language Journal, v. 56, n.3, p. 151158, 1972.

SANTOS, A. A. A. O cloze como técnica de diagnóstico e remediação da compreensão em leitura. Interação em Psicologia, v. 8, n. 2, p. 217-226, 2004.

SANTOS, A. A. A. ; PRIMI, R.; TAXA, F. O. S.; VENDRAMINI, C. M. M. O teste cloze na avaliação da compreensão em leitura. Psicologia: Reflexão e Crítica, v. 15, n. 3, p. 549-560, 2002.

SANTOS, A. A. A.; BORUCHOVITCH, E; OLIVEIRA, K. L . Cloze: um instrumento de diagnóstico e intervenção. São Paulo: Casa do Psicólogo, 2009.

SANTOS, E. C. P. Compreensão de leitura: aplicação da técnica de cloze em estudantes universitários. Intellectus: Revista Acadêmica Digital das Faculdades Unopec, n. 4, p. $58-81,2005$.

SILVA, M. J. M.; SANTOS, A. A. A. A avaliação da compreensão em leitura e o desempenho acadêmico de universitários. Psicologia em Estudo, v. 9, n. 3, p. 459-467. 2004.

SILVA, E. M. T.; WITTER, G. P. Compreensão de texto e desempenho acadêmico em estudantes de psicologia. Estudos de Psicologia, v. 25, n. 3. 2008.

Recebido em: 12/03/2014

Aceito em: 15/04/2014 